\title{
Characteristics of Filtered Neutron Beam Energy Spectra at Dalat Reactor
}

\author{
Tran Tuan Anh'1, Pham Ngoc Son'1, Vuong Huu Tan², Pham Dinh Khang1, Phu Chi Hoa ${ }^{3}$ \\ ${ }^{1}$ Nuclear Research Institute, Dalat, Vietnam \\ ${ }^{2}$ Vietnam Agency for Radiation and Nuclear Safety, Hanoi, Vietnam \\ ${ }^{3}$ The University of Dalat, Dalat, Vietnam \\ Email: pnson.nri@gmail.com
}

Received 9 January 2014; revised 11 February 2014; accepted 6 March 2014

Copyright (C) 2014 by authors and Scientific Research Publishing Inc.

This work is licensed under the Creative Commons Attribution International License (CC BY).

http://creativecommons.org/licenses/by/4.0/

(c) †) Open Access

\begin{abstract}
Filtered neutron technique was applied for producing quasi-monoenergetic neutron beams of 24 $\mathrm{keV}, 54 \mathrm{keV}, 133 \mathrm{keV}$ and $148 \mathrm{keV}$ at the horizontal neutron channel No. 4 of the Dalat Nuclear Research Reactor. The study on physical characteristics of these beam lines has been carried out for efficient applications in neutron capture experiments. The filtered neutron spectrum of each beam has been simulated by Monte-Carlo method and experimentally measured by a gas-filled protonrecoil spectrometer. The neutron fluxes of these filtered beams were measured by the activation technique with standard foils of ${ }^{197} \mathrm{Au}$, using a high efficient HPGe digital gamma-ray spectrometer.
\end{abstract}

\section{Keywords}

\section{Neutron Filter, Proton-Recoil}

\section{Introduction}

The neutron transmission neutron filters technique [1] has been successfully applied to produce quasi-monoenergetic neutrons for basic research and application. Some advantage uses of reactor based filtered beams can be listed as neutron total and capture reaction cross section measurements, calibration of neutron detector, prompt gamma-ray neutron activation analysis (PGNAA) and Boron neutron capture therapy (BNCT).

The Dalat research reactor (DRR), was originally a TRIGA MARK II reactor with a nominal power of 250 $\mathrm{kW}$ completed construction and reached critical state in 1963. The reactor then has been upgraded to nominal power of $500 \mathrm{~kW}$ since 1984. There are three radial and one tangential neutron beam ports at DRR, each of which penetrates the concrete shield structure and the reactor water to provide external beams of neutron origin- 
nated from reactor core [2]. The filtered neutron technique has been introduced at the horizontal channel No. 4 in order to create mono-energetic neutron beams of thermal, $24 \mathrm{keV}, 54 \mathrm{keV}, 59 \mathrm{keV}, 133 \mathrm{keV}$ and $148 \mathrm{keV}$. These filtered neutron beams have been used for implementation of neutron total and radiative capture reaction cross sections measurements, and elemental analysis with PGNAA method.

\section{Filtered Neutron Spectrum Calculations}

The phenomenon of neutron filtration is conditioned by existence in the total neutron cross sections for some atomic nuclei of deep minimums which are the result of interference between the coherent waves of resonance and potential neutron scattering in these nuclei. Therefore, the white neutron spectrum from reactor transmitted such filter components becomes mono-energetic neutrons [1]. The neutron spectrum can be calculated by the following expressions:

$$
\begin{gathered}
\Phi(E)=\Phi_{0}(E) * \exp \left(-\sum_{k} \rho_{k} d_{k} \sigma_{t}(E)\right), \\
I=\frac{\int_{E_{l}}^{E_{h}} \Phi(E) \mathrm{d} E}{\int_{0}^{\infty} \Phi(E) \mathrm{d} E}
\end{gathered}
$$

where $\Phi_{0}(E)$ and $\Phi(E)$ are the energy dependent neutron spectra before and after neutron filters; $\rho_{k}, d_{k}$ and $\sigma_{t}(E)$ are the density, length and total cross section of $k^{\text {th }}$ filter component; $I$ is the relative intensity of the main energy filtered neutron beam [3]. For pre-computation of filtered neutron spectrum with the expression (1) and (2), a computer code named CFNB (Calculation for Filtered Neutron Beams) has been developed for calculation of transmission neutron spectra after filter components. This computer code was evaluated by making comparisons of calculated neutron spectra with corresponding results from Monte-Carlo simulation used the MCNP5 code [4]. The comparison results present relatively agreement between the two calculation methods within statistical uncertainties. The calculated characteristic parameters of dominant neutron energy, resolution, peak neutron flux, and filter materials corresponding for each beam of $24 \mathrm{keV}, 54 \mathrm{keV}, 133 \mathrm{keV}$ and $148 \mathrm{keV}$ are given in Table 1. The results of computed neutron spectra are shown in Figures 1-4.

\section{Filtered Neutron Spectrum Measurements}

The filter components given in Table 1 were successively installed into the horizontal channel No. 4 of the Dalat research reactor, and the corresponding tailored neutron energy spectra of $24 \mathrm{keV}, 54 \mathrm{keV}, 133 \mathrm{keV}$ and 148 $\mathrm{keV}$ beams were measured by using a proton-recoil proportional spectrometer. The measured proton-recoil pulse-high response functions were subtracted by background spectrum which was determined by intercepting the neutron beam with a $10 \mathrm{~cm}$ long polyethylene mixed $\mathrm{B}_{4} \mathrm{C}$ absorber. For mono-energetic neutrons of energy, $E_{n}<10 \mathrm{MeV}$, the recoil-proton response function has a uniform energy distribution. The probability that a recoil-

\begin{tabular}{|c|c|c|c|c|c|c|}
\hline \multirow{2}{*}{$\begin{array}{c}E_{n} \\
(\mathrm{keV})\end{array}$} & \multirow{2}{*}{$\begin{array}{c}\Delta E_{n} \\
(\mathrm{keV})\end{array}$} & \multicolumn{2}{|c|}{ MCNP } & \multicolumn{2}{|c|}{ CFNB } & \multirow[b]{2}{*}{ Filter components } \\
\hline & & $\begin{array}{l}\text { Flux } \times 10^{5} \\
\left(\mathrm{n} \cdot \mathrm{cm}^{-2} \cdot \mathrm{s}^{-1}\right)\end{array}$ & $I(\%)$ & $\begin{array}{l}\text { Flux } \times 10^{5} \\
\left(\mathrm{n} \cdot \mathrm{cm}^{-2} \cdot \mathrm{s}^{-1}\right)\end{array}$ & $I(\%)$ & \\
\hline 24 & 1.8 & $6.2 \pm 0.02$ & 98.3 & 6.2 & 96.7 & $0.2 \mathrm{~g} / \mathrm{cm}^{2}{ }^{10} \mathrm{~B}+20 \mathrm{~cm} \mathrm{Fe}+30 \mathrm{~cm} \mathrm{Al}+35 \mathrm{~g} / \mathrm{cm}^{2} \mathrm{~S}$ \\
\hline 54 & 1.5 & $6.9 \pm 0.01$ & 76.9 & 6.8 & 78.1 & $0.2 \mathrm{~g} / \mathrm{cm}^{2}{ }^{10} \mathrm{~B}+98 \mathrm{~cm} \mathrm{Si}+35 \mathrm{~g} / \mathrm{cm}^{2} \mathrm{~S}$ \\
\hline 133 & 2.7 & $3.3 \pm 0.01$ & 92.9 & 3.1 & 92.3 & $0.2 \mathrm{~g} / \mathrm{cm}^{2}{ }^{10} \mathrm{~B}+50 \mathrm{~g} / \mathrm{cm}^{2} \mathrm{Cr}+10 \mathrm{~cm} \mathrm{Ni}+60 \mathrm{~cm} \mathrm{Si}$ \\
\hline 148 & 14.8 & $40.5 \pm 0.03$ & 94.6 & 41.2 & 95.8 & $0.2 \mathrm{~g} / \mathrm{cm}^{2}{ }^{10} \mathrm{~B}+98 \mathrm{~cm} \mathrm{Si}+1 \mathrm{~cm} \mathrm{Ti}$ \\
\hline
\end{tabular}
proton will have energies between $E_{p}$ and $E_{p}+d E_{p}$ can be evaluated with the following equation [5]. 


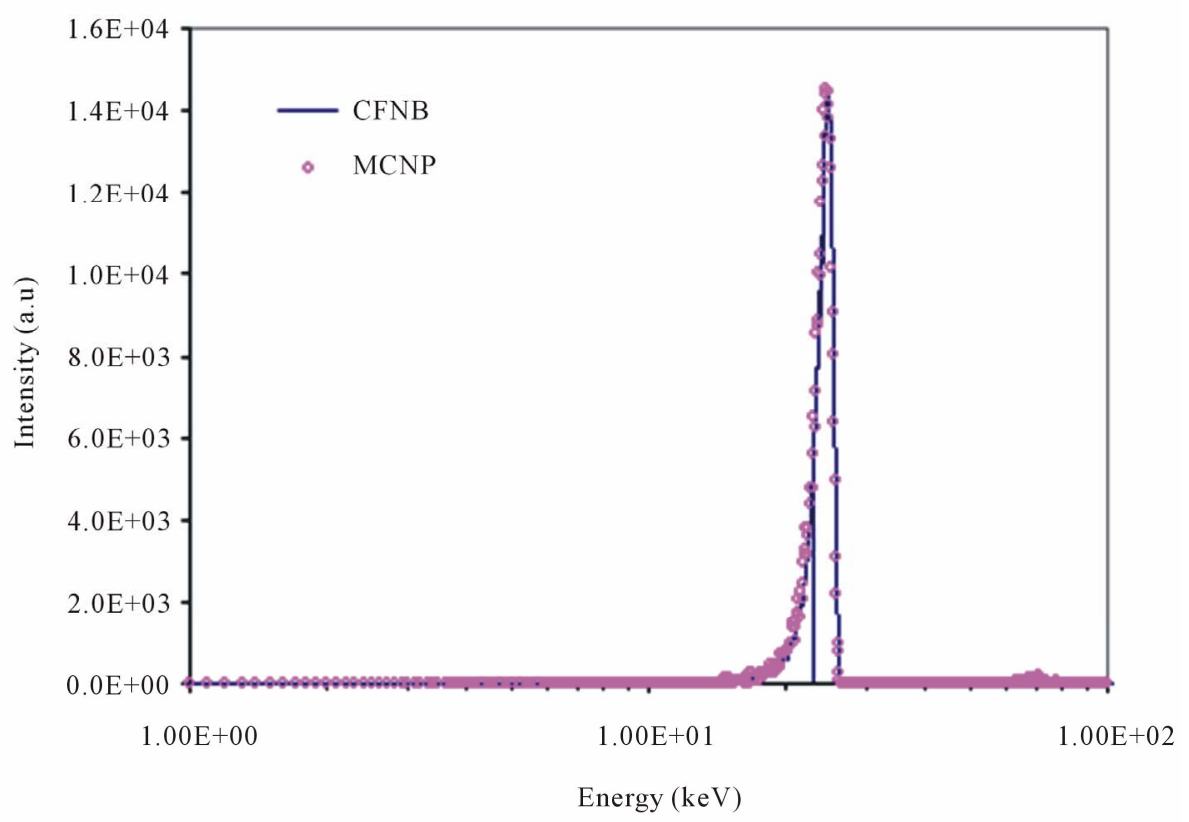

Figure 1. Calculated neutron spectrum of $24 \mathrm{keV}$.

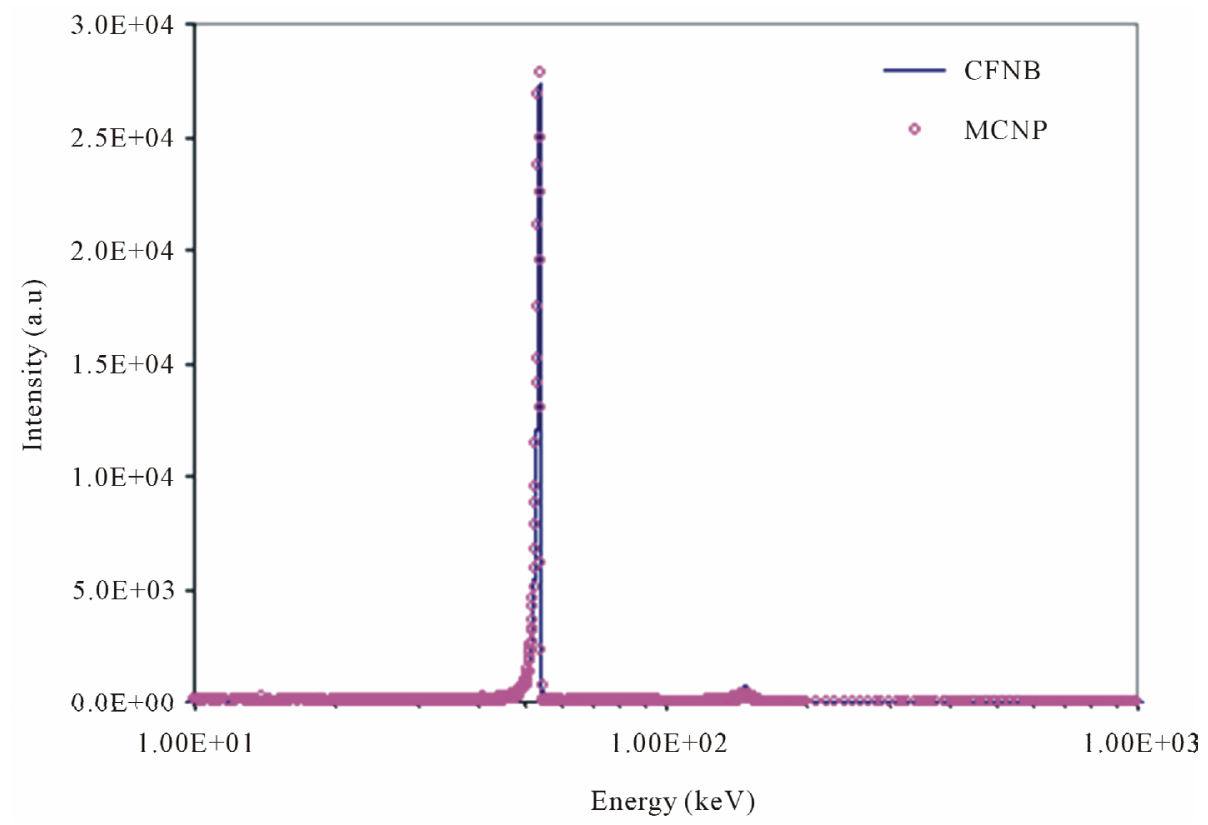

Figure 2. Calculated neutron spectrum of $54 \mathrm{keV}$.

$$
f\left(E_{p}\right) d E_{p}= \begin{cases}\frac{d E_{p}}{E_{n}} & 0 \leq E_{p} \leq E_{n} \\ 0 & E_{p}>E_{n}\end{cases}
$$

Therefore, the response of a proton-recoil counter has a rectangular shape. This simple shape makes it relatively easy to determine the neutron-energy spectrum using the slope of the recoiled proton energy distribution. The proton-recoil response function $f\left(E_{p}\right)$ is related to the neutron energy spectrum $\Phi(E)$ as the following differential equation [5]: 


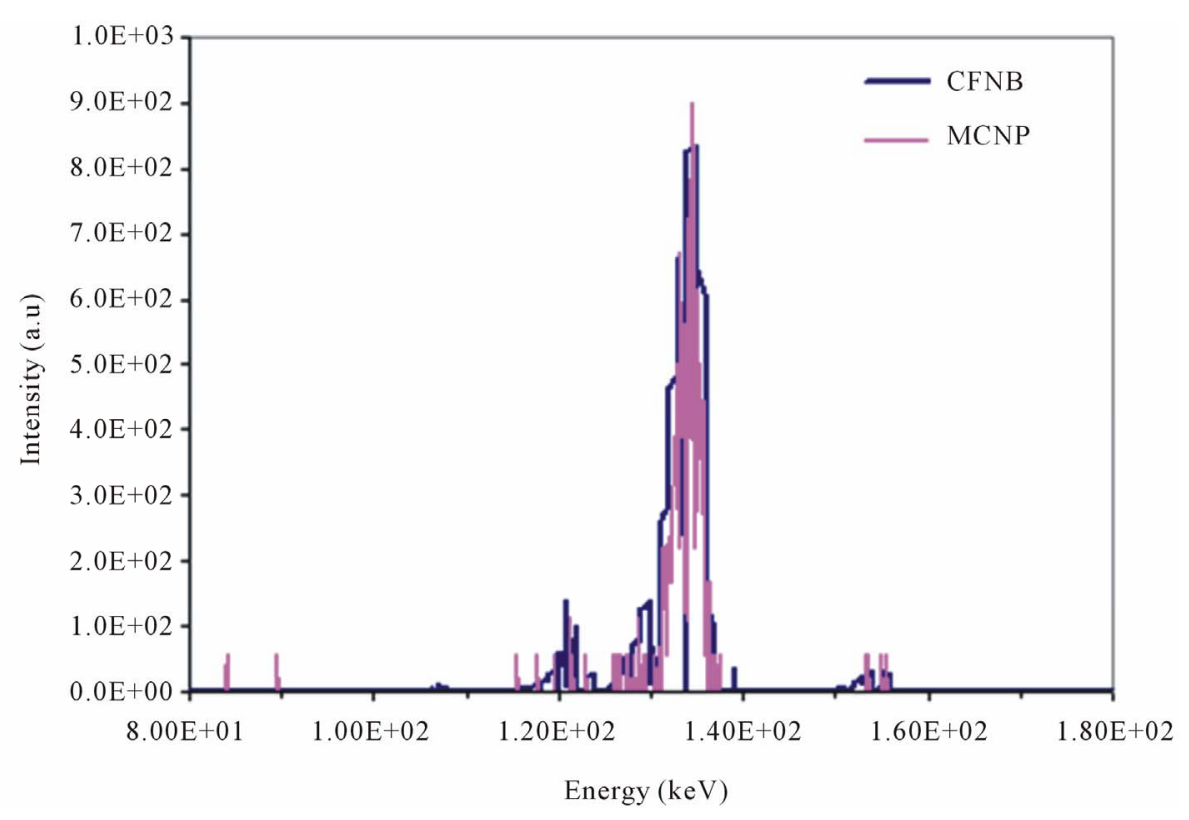

Figure 3. Calculated neutron spectrum of $133 \mathrm{keV}$.

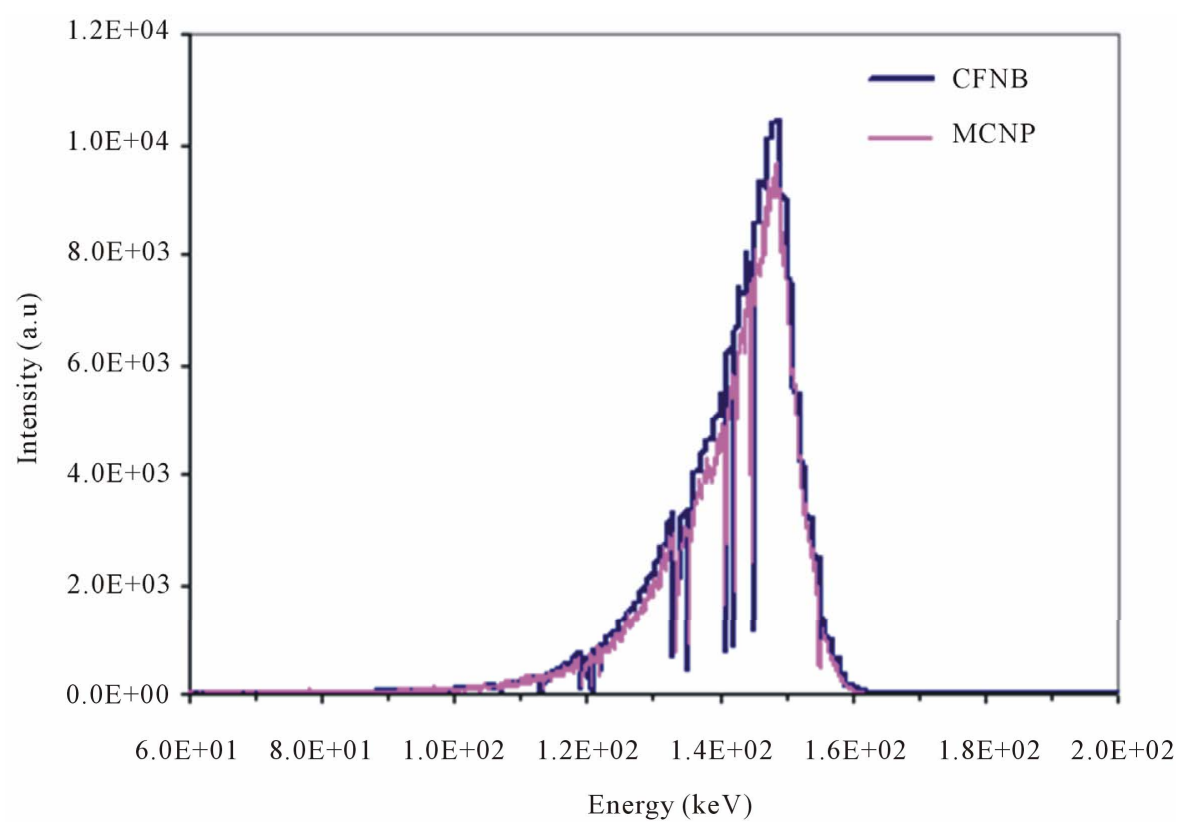

Figure 4. Calculated neutron spectrum of $148 \mathrm{keV}$.

$$
\Phi\left(E_{n}\right)=-\frac{1}{N T} \frac{E_{n}}{\sigma\left(E_{n}\right)}\left|\frac{\mathrm{d} f\left(E_{p}\right)}{\mathrm{d} E_{p}}\right|_{E_{p}=E_{n}}
$$

where $\sigma\left(E_{n}\right)$ is the neutron-proton scattering cross section; $N$ is the number of the hydrogen atom in the effective counter volume; and $T$ is the measuring time. Thus, the neutron spectrum $\Phi(E)$ would be derived from the differential function of the measured proton-recoil data $f\left(E_{p}\right)$. The proton-recoil proportional spectrometer used in these measurements is consisting of a proton-recoil counter model LND-281 (gas filling: $\mathrm{N}+$ $\mathrm{CH}_{4}+\mathrm{N}_{2}$; pressure of 3240 Torr), a preamplifier model PC-142 Ortec, and a CANBERRA multiport-MCA Genie2000 multi-channel analyzer. The measured proton-recoil response function for the $24 \mathrm{keV}$ filtered neutron 
beam and background spectrum are shown in Figure 5.

\section{Results and Discussion}

The characteristic parameters of neutron energy spectrum, relative intensity, and filter materials have been studied for the $24 \mathrm{keV}, 54 \mathrm{keV}, 133 \mathrm{keV}$ and $148 \mathrm{keV}$ filtered neutron beams at the Dalat research reactor. The energy distributions of tailored neutrons were calculated and normalized by transmission and Monte-Carlo methods, in order for optimal selection of filter materials in a practical limited condition of financial support. The calculations code CFNB is also developed and validated for user to evaluate the relative intensity of the expected filtered neutrons and the impurity neutrons. The absolute neutron flux at sample position was measured by activation method with standard foil of ${ }^{197} \mathrm{Au}$ in a $1 \mathrm{~mm}$ thick $\mathrm{Cd}$ cover. The measured values of flux were $6.1 \times 10^{5}, 6.7 \times 10^{5}, 3.2 \times 10^{5}, 3.9 \times 10^{6} \mathrm{n} / \mathrm{cm}^{2} / \mathrm{s}$ for the $24,54,133$ and $148 \mathrm{keV}$ neutron beams, respectively. The results of experimental neutron fluxes are consistent with calculated values, within statistical uncertainties. The proton-recoil response functions and differential neutron spectra of 54, 133 and $148 \mathrm{keV}$ filtered neutron beams are shown in Figures 6-8.

\section{Conclusion}

The study on determination of characteristic parameters has been performed for filtered neutron beams at the

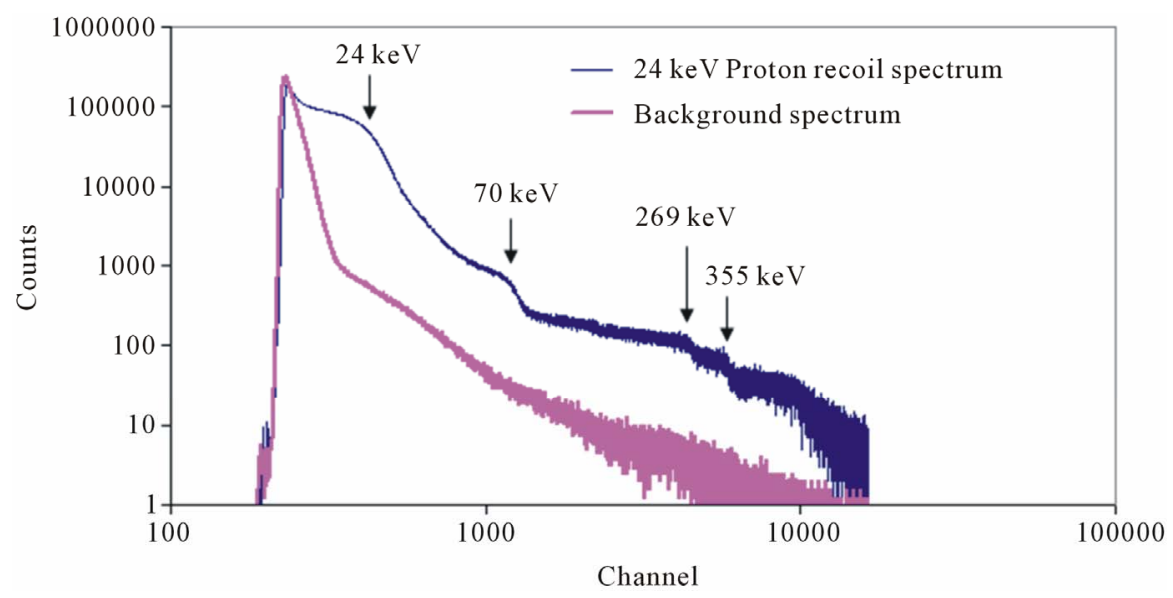

Figure 5. The proton-recoil response function of the $24 \mathrm{keV}$ filtered neutron beam.

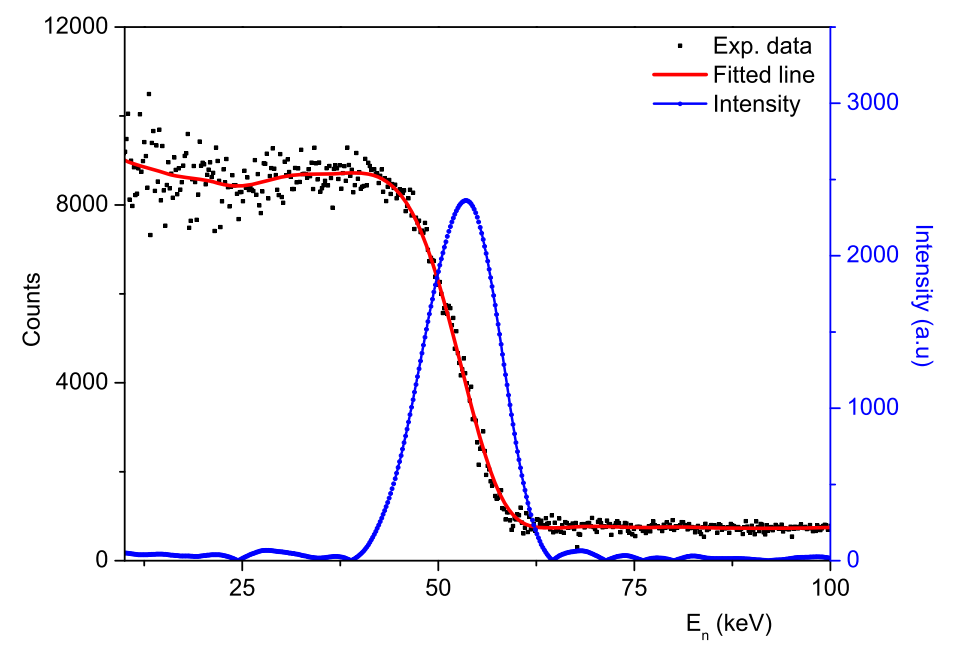

Figure 6. The differential neutron energy spectrum of the $54 \mathrm{keV}$ filtered neutron beam. 


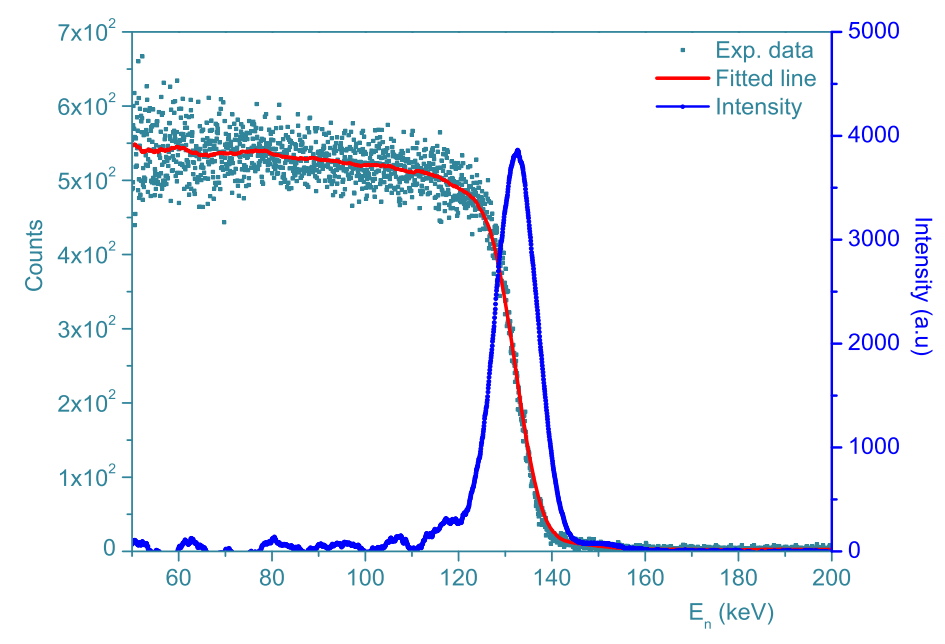

Figure 7. The differential neutron energy spectrum of the $133 \mathrm{keV}$ filtered neutron beam.

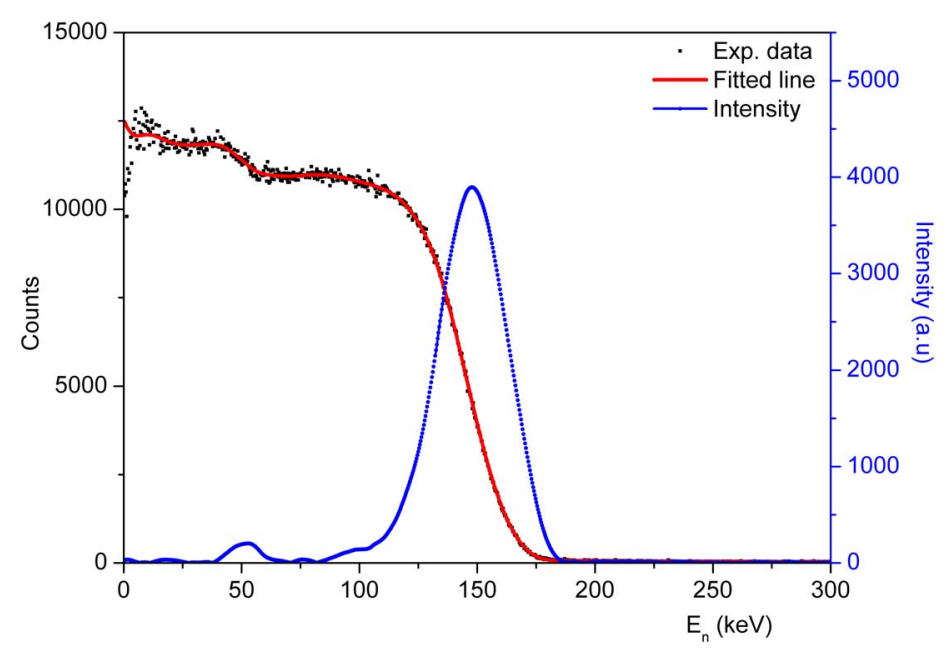

Figure 8. The differential neutron energy spectrum of the $148 \mathrm{keV}$ filtered neutron beam.

horizontal channel No. 4 of Dalat research reactor. The neutron filter technique has been effectively applied to providing mono-energetic neutron beam lines with qualified characteristics for basic research and related applications at the Dalat Nuclear Research Institute. These neutron beams can be used as mono-energetic neutron sources for measurements of neutron capture and total reaction cross sections. The applications of these monoenergetic neutron beams are also proposed for energy dependent calibration of neutron detectors.

\section{Acknowledgements}

The authors are grateful to the director board of Dalat Nuclear Research Institute for their great encouragement and helpful in creating an advantage research condition. This research is funded by Vietnam National Foundation for Science and Technology Development (NAFOSTED) under grant number “103.04-2012.59".

\section{References}

[1] Simpson, O.D. and Miller, L.G. (1968) A Technique to Measure Neutron cross Sections in the Low keV Energy Region. Nuclear Instruments and Methods, 61, 245-250. http://dx.doi.org/10.1016/0029-554X(68)90236-X

[2] General Atomic (1961) TRIGA Mark II Reactor General Specifications and Description. GA-2627, Division of General Dynamics, USA. 
[3] Gritzay, O.O., Kolotyi, V.V. and Kaltchenko, O.I. (2001) Neutron Filters at Kyiv Research Reactor. National Academy of Sciences of Ukraine, Institute for Nuclear Research, Preprint KINR-01-6, Kiev.

[4] X-5 Monte Carlo Team (2005) MCNP_A General N-Particle Transport Code, Version 5. Volume I: Overview and Theory, LA-UR-03-1987, Los Alamos National Laboratory, USA.

[5] Verbinski, V.V. and Giovanni, R. (1974) Proportional Counter Characteristics and Applications to Reactor Neutron Spectrometry. Nuclear Instruments and Methods, 114, 205-231. http://dx.doi.org/10.1016/0029-554X(74)90536-9 